\title{
Expression of Cellular Receptors in the Ischemic Hemisphere of Mice with Increased Glucose Uptake
}

\author{
Jin Soo Lee ${ }^{1,2, *}$, Ji Man Hong ${ }^{1,2}$, Bok Seon Yoon ${ }^{1,2}$, Keoung Sun Son ${ }^{1,2}$, Kyung Eon Lee ${ }^{3}$, \\ Doo Soon Im ${ }^{4}$, Bok-Nam Park ${ }^{5}$, Young-Sil An ${ }^{5}$, Dong Hoon Hwang ${ }^{6}$, Chan Bae Park ${ }^{7}$, \\ Byung Gon Kim ${ }^{2,6}$ and Eun-hye Joe ${ }^{2,6,8}$
}

${ }^{1}$ Department of Neurology, Ajou University School of Medicine, ${ }^{2}$ Neuroscience Graduate Program, Department of Biomedical Sciences, Ajou University School of Medicine, Suwon 16499, ${ }^{3}$ School of Pharmacy, Kyung Hee University, Seoul 02447, Korea,

${ }^{4}$ Department of Cellular \& Molecular Medicine, University of Ottawa, Ottawa, ON K1H 8M5, Canada, ${ }^{5}$ Department of Nuclear Medicine and Molecular Imaging, Ajou University School of Medicine, ${ }^{6}$ Department of Brain Science, Ajou University School of Medicine, ${ }^{7}$ Department of Biology, Ajou University School of Medicine, ${ }^{8}$ Department of Pharmacology, Ajou University School of Medicine, Suwon 16499, Korea

Many previous studies have shown reduced glucose uptake in the ischemic brain. In contrast, in a permanent unilateral common carotid artery occlusion (UCCAO) mouse model, our pilot experiments using 18F-fluorodeoxyglucose positron emission tomography (FDG PET) revealed that a subset of mice exhibited conspicuously high uptake of glucose in the ipsilateral hemisphere at 1 week post-occlusion (asymmetric group), whereas other mice showed symmetric uptake in both hemispheres (symmetric group). Thus, we aimed to understand the discrepancy between the two groups. Cerebral blood flow and histological/metabolic changes were analyzed using laser Doppler flowmetry and immunohistochemistry/Western blotting, respectively. Contrary to the increased glucose uptake observed in the ischemic cerebral hemisphere on FDG PET ( $\mathrm{p}<0.001$ ), cerebral blood flow tended to be lower in the asymmetric group than in the symmetric group (right to left ratio [\%], $36.4 \pm 21.8$ vs. 58.0 \pm 24.8 , $p=0.059$ ). Neuronal death was observed only in the ischemic hemisphere of the asymmetric group. In contrast, astrocytes were more activated in the asymmetric group than in the symmetric group $(\mathrm{p}<0.05)$. Glucose transporter-1, and monocarboxylate transporter- 1 were also upregulated in the asymmetric group, compared with the symmetric group ( $\mathrm{p}<0.05$, respectively). These results suggest that the increased FDG uptake was associated with relatively severe ischemia, and glucose transporter-1 upregulation and astrocyte activation. Glucose metabolism may thus be a compensatory mechanism in the moderately severe ischemic brain.

Key words: Brain ischemia, 4-fluoro-4-deoxyglucose, Positron-emission tomography, Glucose transporter type 1, Astrocytes

\section{INTRODUCTION}

Blockage of blood vessels in the brain causes a reduction in cerebral blood flow (i.e., cerebral ischemia) and can lead to neuronal damage [1]. In the acute phase, oxygen metabolism is well-known

Received September 30, 2019, Revised January 16,2020,

Accepted February 6, 2020

* To whom correspondence should be addressed.

TEL: 82-31-219-5175, FAX: 82-31-219-5178

e-mail:jinsoo22@gmail.com to be altered on positron emission tomography (PET) [2], but glucose metabolism has not been well-defined. Glucose uptake is typically not visible in infarction core lesions because neuronal cells have become necrotic [3, 4]. However, when ischemia is sustained for several days, compensatory mechanisms may develop to offset its effects; these may include hyperperfusion due to collateral circulation and recanalization, or increased metabolism to supply energy to parenchymal cells [5-8]. Increased glucose uptake should be cautiously interpreted because it could be influenced by reperfusion phenomena in the ischemic brain [9].

Unilateral common carotid artery occlusion (UCCAO) has been
Copyright $\odot$ Experimental Neurobiology 2020. www.enjournal.org
This is an Open Access article distributed under the terms of the Creative Commons Attribution Non-Commercial License (http://creativecommons.org/licenses/by-nc/4.0) which permits unrestricted non-commercial use, distribution, and reproduction in any medium, provided the original work is properly cited. 
used to generate a mouse model with mild unilateral cerebral ischemia without recanalization. In this model, vascular cognitive impairment is induced [10], although cerebral blood flow (CBF) exhibits a mild decrease of 20 30\% and cellular necrosis does not frequently occur [11]. Furthermore, in an animal model of Alzheimer's disease, UCCAO-induced cognitive decline was reported to be additive or synergistic [12]. Because ${ }^{18} \mathrm{~F}$-fluorodeoxyglucose (FDG) PET revealed metabolic deficits at 4 and 8 weeks after UCCAO [12], cognitive decline may be related to metabolic deficits. In brain diseases, metabolic changes may occur prior to structural and anatomical changes; therefore, FDG PET imaging has been widely used as a complementary addition to conventional imaging methods to evaluate degenerative neurologic disease in humans and in animal models [13-15]. Changes in FDG PET activity, however, can include several features, such as neural activity, neural energy metabolism, and cerebral blood flow [3, 16, 17]. These factors warrant further evaluation to determine a causative mechanism.

In our pilot experiments, we observed asymmetric uptake of FDG in a subset of mice on animal PET scans 7 days after UCCAO. While recanalization-related auxiliary perfusion, which may affect high glucose uptake, can develop in humans [18], recanalization was not induced in our model of UCCAO. Thus, we hypothesized that this phenomenon might reflect increased glucose metabolism in the ischemic brain hemisphere, rather than hyperperfusion or increased neural activity. To test this hypothesis, we evaluated cerebral perfusion status and performed histological and immunological studies to observe metabolic changes.

\section{MATERIALS AND METHODS}

\section{Animal setting}

Experiments were performed using male C57BL/6 mice (weight 25 30 g) obtained from Orient Bio (Gyunggi-do, South Korea). Eight-week-old mice were used for quantitative analyses. Animal protocols were approved by the Laboratory Animal Research Center of Ajou University School of Medicine. Mice were kept in cages which were maintained at a constant temperature $\left(22 \pm 2^{\circ} \mathrm{C}\right)$ and humidity $(55 \pm 10 \%)$ under light-controlled conditions (lights on from 7:00 to 19:00 h), with ad libitum access to food and water.

Animals were anesthetized with a mixture of $2 \%$ isoflurane in $70 \% \mathrm{~N}_{2} \mathrm{O}$ and $30 \% \mathrm{O}_{2}$, using a Fluotec vaporizer. For the UCCAO model, the skin was incised at the anterior midline of the neck; the right common carotid artery was then exposed and ligated doubly with a 6-0 silk, ensuring separation from the vagus nerve. Separate groups of sham-operated mice underwent the same surgery except without ligation. Rectal temperature was monitored during anes- thesia and was maintained at $37.0 \pm 0.5^{\circ} \mathrm{C}$ by an automated heating pad connected to the rectal probe (Animal Blanket Controller, CMA8003761, Exacon Denmark, Germany). Thereafter, animals were housed with ad libitum access to food and water.

Seven days after UCCAO, mice were intraperitoneally anesthetized with $10 \%$ chloral hydrate, then transcardially perfused with $4 \%$ paraformaldehyde (PFA) in phosphate-buffered saline (PBS). The brains were removed and post fixed for $\geq 24 \mathrm{~h}$ in $4 \%$ PFA at $4{ }^{\circ} \mathrm{C}$, then dehydrated with $30 \%$ sucrose. The tissues were cut using a cryostat machine (CM3050S, Leica, Germany) to generate 20- $\mu \mathrm{m}$ thick coronal sections, then stored in stock solution at $4{ }^{\circ} \mathrm{C}$.

\section{${ }^{18}$ F-fluorodeoxyglucose positron emission tomography for animal}

Regional glucose metabolism was evaluated using FDG PET imaging. Mice were anesthetized with chloral hydrate $(400 \mathrm{mg}$ / $\mathrm{kg}$, i.p.) and intravenously injected with $55.5 \mathrm{MBq}$ of ${ }^{18} \mathrm{~F}$-FDG through the tail vein. After an uptake period of 60 minutes, animals were placed in a spread prone position and scanned with the eXplore Vista (GE Healthcare, Milwaukee, WI), which consisted of an 11.8 -cm diameter ring with 36 position-sensitive $\gamma$-ray scintillation detectors (dual layer [GSO, LYSO crystal] phoswich detectors), providing a $6.7 \mathrm{~cm}$ transaxial and a $4.8 \mathrm{~cm}$ axial field of view, with an image resolution $<1.0 \mathrm{~mm}$. A 40-min static acquisition was performed in the three-dimensional mode, and images were reconstructed by a maximum a posteriori probability algorithm. Corrections for dead time, random, scatter, decay, and normalization were performed. Region of interest covers each supratentorial hemisphere.

\section{Cerebral blood flow (CBF) measurements}

$\mathrm{CBF}$ was measured in each hemisphere of the experimental mice. A moorVMS-LDF ${ }^{\mathrm{TM}}$ laser Doppler blood flow monitor (Moor Instruments, Devon, UK) was used to detect the CBF of mice under inhalation anesthesia. A laser Doppler flowmetry probe was situated on the skull, $1.0 \mathrm{~mm}$ posterior to bregma and $2.5 \mathrm{~mm}$ lateral to the midline.

\section{Cresyl violet staining}

The tissue sections were mounted on slides. Mounted sections were incubated with a $0.1 \%$ cresyl violet solution for 10 -min. Neuronal damage was analyzed by quantifying cell changes in the cortical layer (Supplementary Fig. 1). Survived neurons, in which the cytoplasm is darkly stained with a more lightly stained nucleus, were counted in a $320 \mu \mathrm{m}$ wide strip of cortex from images taken at $400 \times$ magnification using an optical microscope (DM 2000, Leica, Germany). 


\section{Immunohistochemistry}

Brain sections were mounted on slides and washed with PBS. Endogenous peroxidase activity was quenched with $0.3 \%$ hydrogen peroxide for $5 \mathrm{~min}$ and $0.25 \%$ Triton X-100 for $10 \mathrm{~min}$. After blocking in $10 \%$ normal serum for $1 \mathrm{~h}$, the sections were incubated with anti-glucose transporter 1 (GLUT1) (dilution 1:500; Abcam; AB40084), anti-active caspase-3 (dilution 1:20; Chemicon; AB3623), rabbit anti-glucose transporter GLUT3 (dilution 1:500; Abcam; AB1344), rabbit glial fibrillary acidic protein (GFAP) marker (dilution 1:500; Dako; Z0334), guinea pig glutamate transporter-1 (GLT-1) marker (dilution 1:200; Chemicon; AB1783), rabbit monocarboxylate transporter 1 (MCT-1) marker (dilution 1:50; Santa Cruz; SC-50324), rabbit monocarboxylate transporter 2 (MCT-2) marker (dilution 1:200; Millipore; AB33542), or rabbit monocarboxylate transporter 4 (MCT-4) marker (dilution 1:200; Millipore; AB3314p) overnight at $4{ }^{\circ} \mathrm{C}$. After washing, the sections were incubated with biotinylated anti-mouse IgG (dilution 1:500;
Vector; BA-2000) for $2 \mathrm{~h}$ and ABC (PK-6100, Vector) for $1 \mathrm{~h}$; then, diaminobenzidine (DAB, SK-4100, Vector) was used to visualize the signal.

Quantification of the GLUT1 density in the cortex was performed with a microscope (SMZ745T, Nikon) at a magnification of $400 \times$. Relative optical densities were measured as the mean gray values on inverted black-white images. For other markers, after washing, the sections were incubated with AlexaFluor 555 goat anti-rabbit IgG (dilution 1:500; Invitrogen; A21428) or Texas red anti-guinea pig (dilution 1:200; Vector; TI-7000) for $2 \mathrm{~h}$. MCT1 , and -2 were pretreated with an acetic acid:ethanol (1:2 ratio) mixture for $5 \mathrm{~min}$ at $-20^{\circ} \mathrm{C}$ before staining. Quantification of the GLUT3, GLT-1, MCT-1, 2, and -4 densities was performed with a fluorescence microscope (Axiovert 200 M, Zeiss, Germany) at a magnification of $400 \times$, with an AxioCam MR3 camera. Percentages of density were measured as mean density values using NIH ImageJ software; all data were normalized to the value of the con-
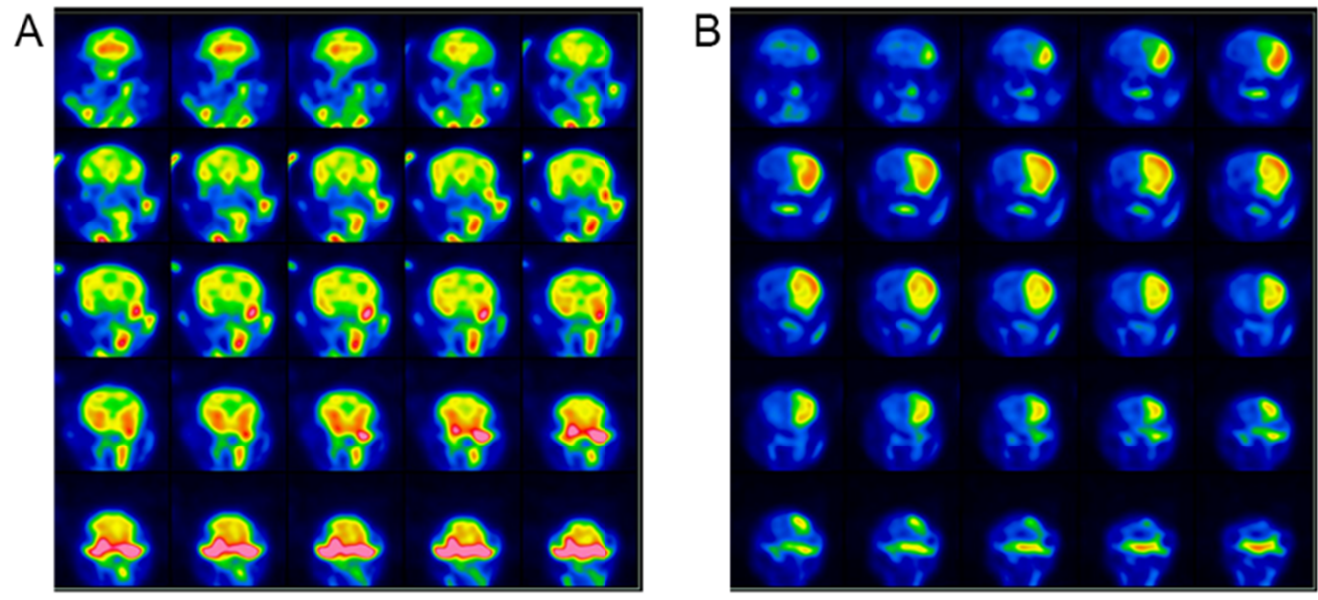

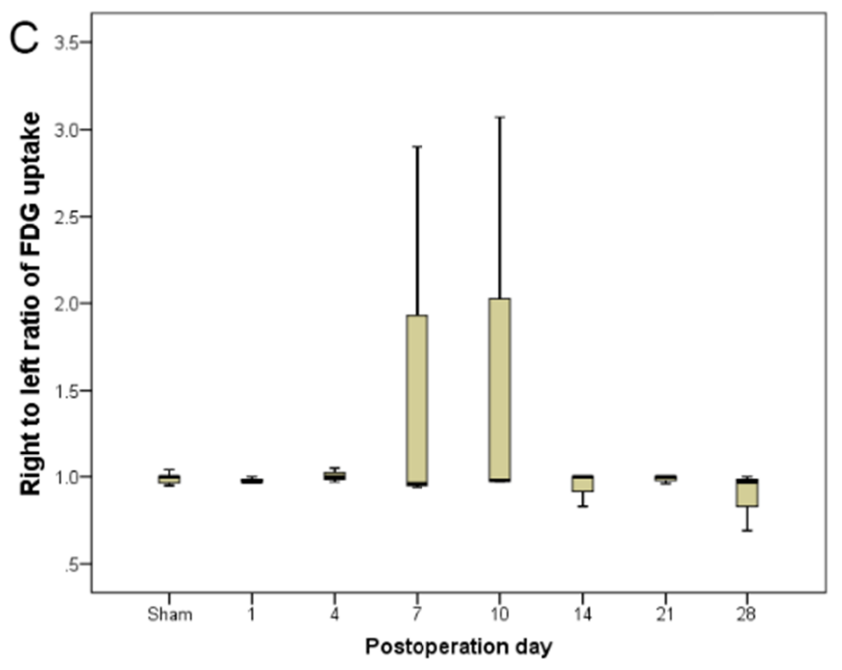

Fig. 1. An incidental observation using 18F-fluorodeoxyglucose positron emission tomography in mice, following unilateral common carotid artery occlusion (UCCAO). In our pilot evaluations, glucose uptake patterns were classified into two types after the operation. (A) An example of a mouse brain with symmetrical uptake despite right UCCAO. In most mice with the UCCAO operation, glucose uptake was symmetrical. (B) An example of a mouse brain with asymmetrical glucose uptake in the ischemic hemisphere (right side on the figure). In a subset of mice, glucose uptake was found to asymmetrically increase in the right hemisphere. (C) To determine the most appropriate time for viewing asymmetric glucose uptake, UCCAO-operated ( $\mathrm{n}=3$ per day) and shamoperated mice ( $\mathrm{n}=1$ per day) were examined at days $1,4,7,10,14,21$, and 28 after the operation. One of three UCCAO mice showed an increase in glucose uptake at 7 and 10 days, respectively. One of three UCCAO mice showed a decrease in glucose uptake at 28 days. Otherwise, symmetric glucose uptake patterns were shown. The seventh day was selected for further glucose uptake evaluations. 
trol. Quantification of active Caspase-3 and GFAP was performed by counting positive cells.

\section{Western blotting}

Cortical tissue was homogenized on ice in a lysis buffer $(50 \mathrm{mM}$ Tris-HCl, pH 7.4, 150 mM NaCl, 1\% Triton X-100, 0.25\% sodium deoxycholate, $0.1 \%$ sodium dodecylsulfate [SDS], 1 mM EDTA, 1\% protease inhibitor cocktail; Sigma). After centrifugation, the supernatant was collected and assayed for protein concentration using the BCA method. Lysate samples, containing $20 \mu \mathrm{g}$ of protein, were fractionated by SDS-polyacrylamide gel electrophoresis and transferred to a nitrocellulose membrane. Then, the membranes were blocked with 10\% non-fat milk in PBS + Tween-20 for $1 \mathrm{~h}$ and incubated with the following primary antibodies: anti-betaactin (dilution 1:5000; Abcam; AB8227), anti-glucose transporter GLUT1 (dilution 1:5000; Abcam; AB40084), rabbit anti-glucose transporter GLUT3 (dilution 1:5000; Abcam; AB1344), or rabbit glial fibrillary acidic protein (GFAP) marker (dilution 1:5000; Dako; Z0334) overnight at $4{ }^{\circ} \mathrm{C}$. After washing, membranes were incubated with goat anti-rabbit IgG HRP (dilution 1:5000; Santa Cruz; SC-2004) and HRP-labeled anti-mouse IgG (dilution 1:2500; Vector; PI-2000) for $2 \mathrm{~h}$ at room temperature. The signals were visualized using an enhanced ECL Western blotting substrate solution (Pierce, 32209). Quantification of the immunoreactive bands was performed by scan imaging, and quantitative measurements were analyzed using the ImageJ program.

\section{Statistics}

Imaging and histological examinations were compared between the right ischemic hemispheres of the asymmetric and symmetric groups. Data are presented as the mean \pm standard error of the mean (SEM). A $t$-test was used to compare continuous values between groups. P-values $<0.05$ were considered to be statistically significant. Statistical analyses were performed using IBM SPSS Statistics version 22 software (IBM Corp., Armonk, NY, USA).

\section{RESULTS}

\section{Appearance of asymmetric FDG uptake in mice after UCCAO}

We used FDG PET to analyze how much brain metabolism decreased upon ischemic insult. Unexpectedly, in our pilot experiments, a subset of mice showed increased FDG uptake in the ipsilateral (right side) hemisphere 7 days after UCCAO (Fig. 1A and 1B). Most UCCAO mice showed a symmetric distribution of FDG uptake (symmetric group) (Fig 1A). In contrast, a small portion of mice exhibited an asymmetric increase in FDG uptake throughout the entire ipsilateral hemisphere (asymmetric group) (Fig. 1B). Interestingly, whereas most mice with UCCAO exhibited a symmetric glucose uptake pattern until 28 days post-surgery, one of the three mice exhibited increased glucose uptake in the ipsilateral hemisphere at days 7 and 10 (Fig. 1B), but not at days 1 or 3. These results raised questions regarding the underlying mechanisms of symmetric and asymmetric FDG uptake and the effects of different patterns of FDG uptake on ischemic injury.

During quantitative analyses of the region of interest centered in each hemisphere on FDG PET, the mean uptake value in the right hemisphere was significantly higher in the asymmetric group than in the symmetric and sham groups ( $\mathrm{p}<0.001$; Fig. $2 \mathrm{~A})$. In contrast, the mean uptake value was not different among the left hemispheres of the asymmetric, symmetric, and sham groups (Fig. 2B).
A

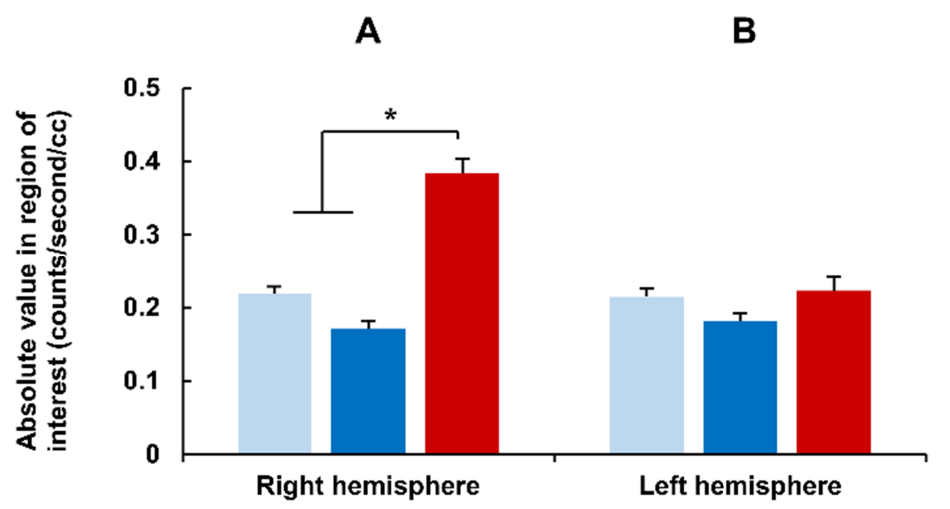

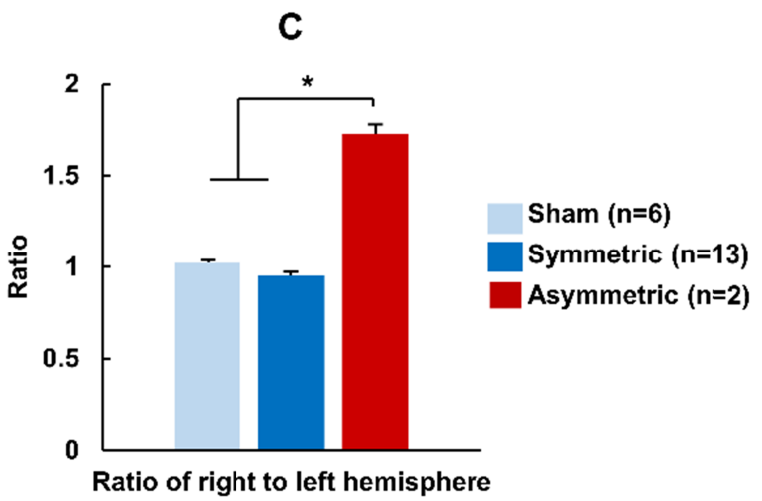

Fig. 2. Absolute value of glucose uptake in the region of interest of each hemisphere for the 18F-fluorodeoxyglucose positron emission tomography scans. Comparisons were made among the unilateral common carotid artery occlusion-operated mice with and without asymmetric uptake, and shamoperated mice. (A) The quantity of glucose uptake in the ipsilateral hemisphere was greater in the asymmetric group than in the other groups. (B) The quantity of glucose uptake in the contralateral hemisphere did not decrease in the asymmetric group, relative to other groups. (C) Finally, the uptake ratio of the right to left hemisphere was higher in the asymmetric group than in the other groups. ${ }^{*} \mathrm{p}<0.001$. 
The mean uptake values in the right and left hemispheres were approximately two-fold higher in the asymmetric group than in the other groups $(\mathrm{p}<0.001$; Fig $2 \mathrm{C})$.

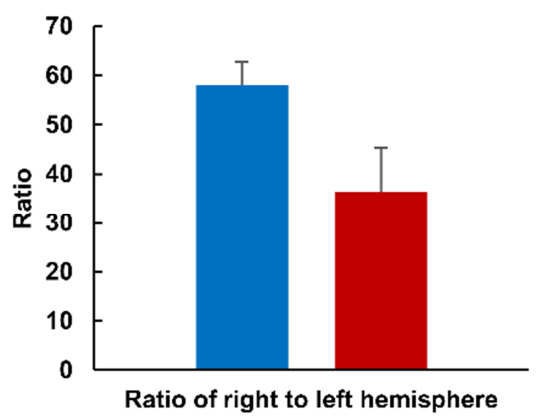

Symmetric $(n=27)$

Asymmetric $(n=6)$

Fig. 3. Comparison of cerebral blood flow in the right hemisphere between the symmetric and asymmetric groups, as measured by laser Doppler flowmetry. Among 33 unilateral common carotid artery occlusion-operated mice whose laser Doppler flowmetry and 18Ffluorodeoxyglucose positron emission tomography scan were both taken at 7 days after the operation, six mice exhibited asymmetric increases in glucose uptake within their right hemispheres (i.e., asymmetric group) while twenty seven mice showed a symmetric pattern (symmetric group). Cerebral perfusion tended to be lower in the ipsilesional hemisphere of the asymmetric group, compared to the symmetric group $(\mathrm{p}=0.059)$.

\section{Reduced cerebral blood flow in the ipsilesional hemisphere} of the asymmetric group

Next, we evaluated whether the increased glucose uptake in FDG PET reflected increased CBF. At 7 days after UCCAO, CBF was measured in each hemisphere using laser Doppler flowmetry. Contrary to the glucose uptake pattern, CBF tended to be lower in the right hemisphere of the asymmetric group than in the symmetric group (ratio of right to left CBF, $36.4 \pm 21.8 \%$ vs. $58.0 \pm 24.8 \%$, $\mathrm{p}=0.059$; Fig. 3). These results suggest that increased glucose uptake after UCCAO in the asymmetric group was not related to blood flow.

\section{Increased neuronal death and expression of glucose/lac-} tate transporters in the ipsilateral hemisphere of the asym-

\section{metric group}

Since blood flow was inversely associated with glucose uptake in the asymmetric UCCAO model, we further examined possible histological differences in symmetric and asymmetric brains of the UCCAO model. First, we examined neuronal damage. Using cresyl violet and caspase- 3 staining, neuronal damage was barely detectable in the symmetric group (Fig. 4). However, in the asymmetric group, widespread neuronal necrosis and apoptosis were observed
A

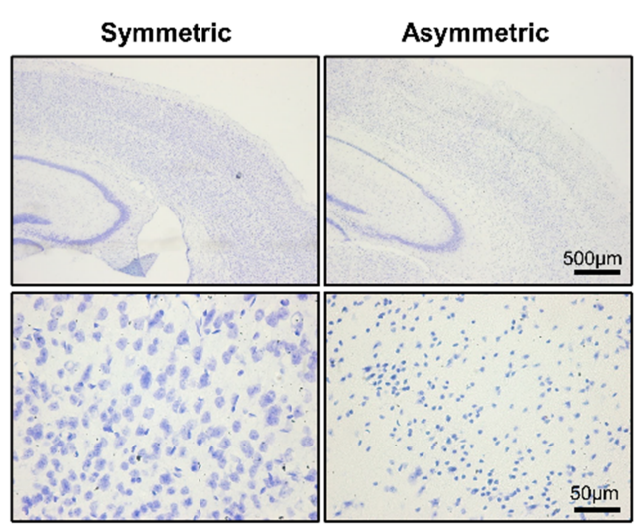

C

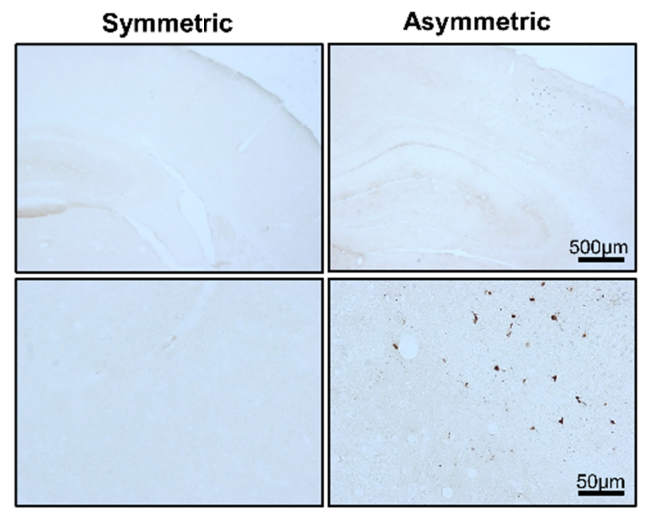

B

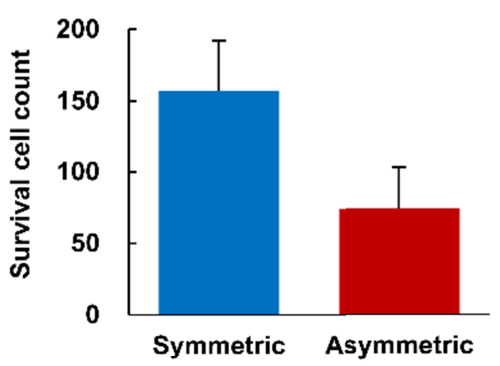

D

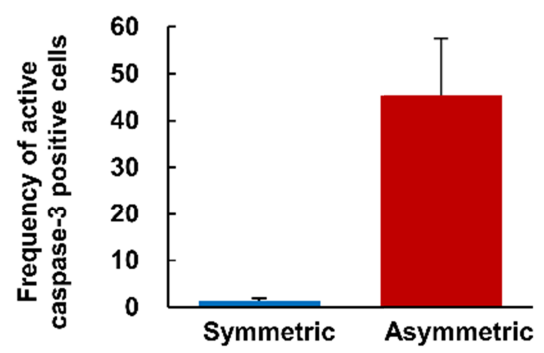

Fig. 4. Comparison of histological changes between symmetric and asymmetric groups. Neuronal damage was not seen in any mouse within the symmetric group. However, the asymmetric group showed evidence of neuronal necrosis and apoptosis using cresyl violet and caspase- 3 staining, respectively. (A) Cresyl violet staining in the cortex of the mouse brain. (B) The number of neuronal cells that survived tended to decrease in the ischemic hemisphere of the asymmetric group compared to symmetric group; however, this was not statistically significant $(\mathrm{p}=0.186)$. (C) Immunohistochemistry of active caspase- 3 in the cortex of the ischemic hemisphere. (D) Apoptotic cells were frequently observed in the ischemic hemisphere of the asymmetric group compared to the symmetric group; however, this was not statistically significant $(\mathrm{p}=0.177)$. Data are expressed as the mean \pm standard error of the mean ( $\mathrm{n}=6 /$ group). 
A

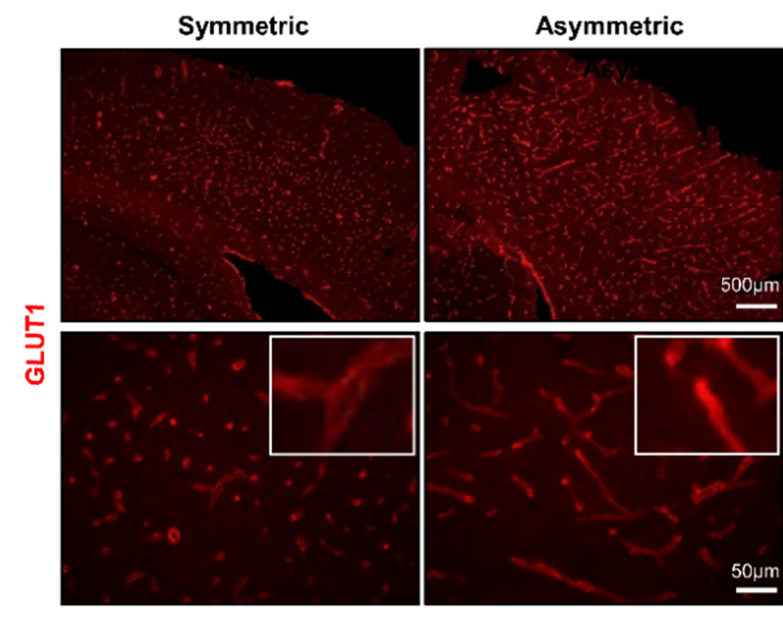

E

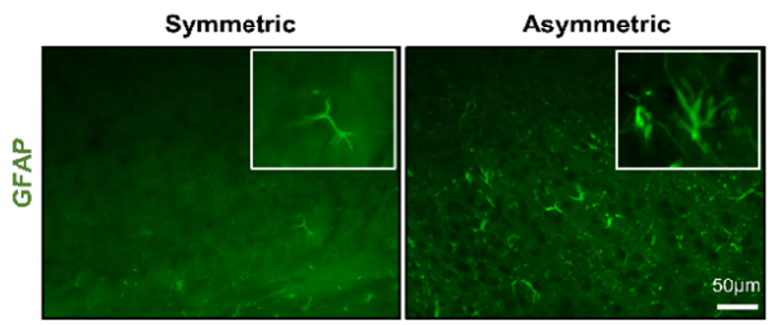

H

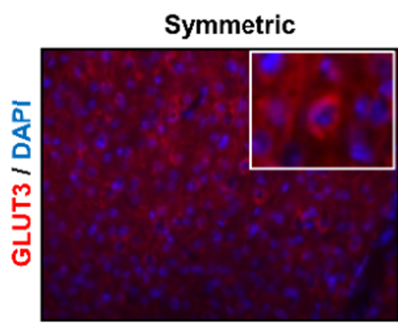

Asymmetric

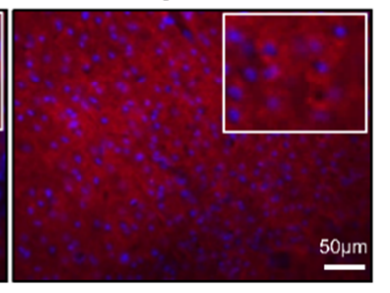

B
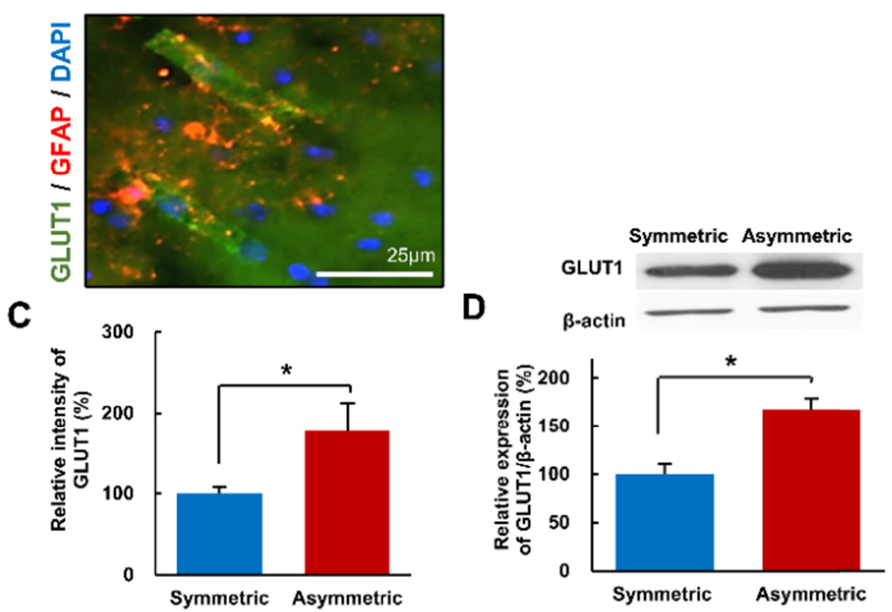

F
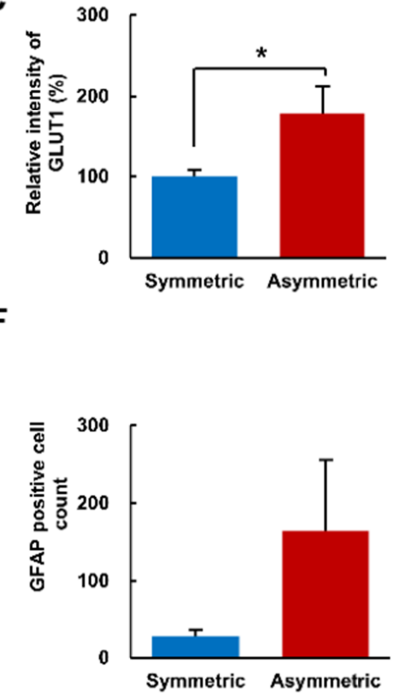

I

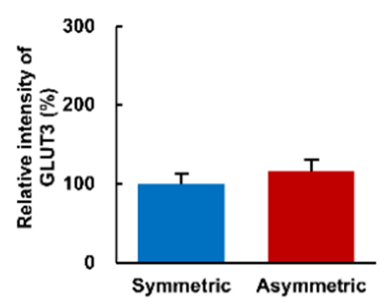

G

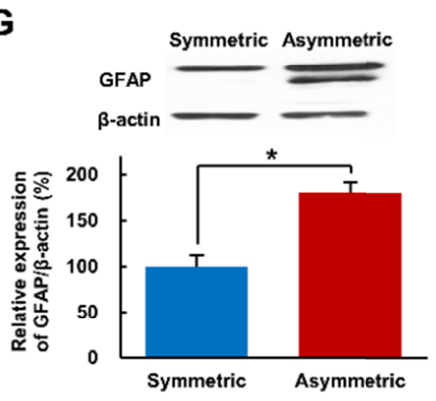

J

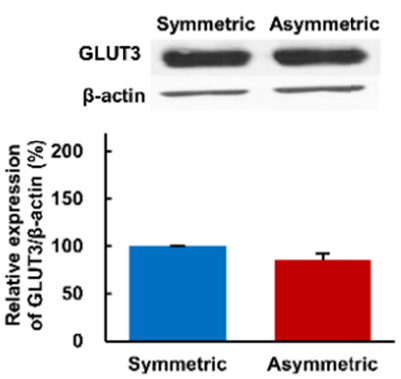

Fig. 5. Expression of GLUT1, GLUT3, and GFAP in the ipsilesional cortex of the mouse brain with right UCCAO. (A) An increase in the immunohistochemical expression of GLUT1 (red) was observed in the vessels of the asymmetric group, compared with the symmetric group. (B) Double immunofluorescence staining of GLUT1 and GFAP. GLUT1 along the vessels showed co-localization with an astrocytic foot process in the asymmetric group. (C) Intensity measurement to quantify the GLUT1 level showed a significant increase in the asymmetric group ( $n=6 /$ group, $p<0.05$ ). (D) Western blot analysis of GLUT1 ( $55 \mathrm{kDa})$ in the ischemic hemisphere of the mouse brain. Quantitation of GLUT1 expression was performed, relative to actin expression. The GLUT1 level was significantly increased in the asymmetric group compared to the symmetric group ( $\mathrm{n}=3 /$ group, $\mathrm{p}<0.05$ ). (E) Immunohistochemical expression of GFAP (green), indicating astrocyte activation, was observed to increase in the asymmetric group. (F) GFAP-positive cells were more frequently observed in the right hemisphere of the asymmetric group than in the symmetric group, however, this difference was not statistically significant ( $\mathrm{n}=6$ /group). (G) Western blot analysis of GFAP (55k Da) shows significantly increased expression in the right cortex of the asymmetric group compared to the symmetric group ( $\mathrm{n}=3$ /group, $\mathrm{p}<0.05$ ). (H) GLUT3 (red) expression was faintly observed in both groups. (I) Intensity of GLUT3 did not differ between the two groups ( $\mathrm{n}=6 /$ group). (J) Western blot analysis of GLUT3 ( $54 \mathrm{kDa}$ ) showed no difference between the two groups ( $\mathrm{n}=3$ / group). Data are expressed as the mean \pm standard error of the mean. GLUT, anti-glucose transporter; GFAP, glial fibrillary acidic protein. ${ }^{*} \mathrm{p}<0.05$ ( $t$-test).

(Fig. 4) within the right hemisphere, but not in the contralateral hemisphere.

Next, we examined the expression levels of glucose transport- ers, such as GLUT1 and GLUT3, as underlying mechanisms of asymmetric FDG uptake in mice after UCCAO. GLUT1, which appeared to be highly expressed along the vessels and co-localized 
A

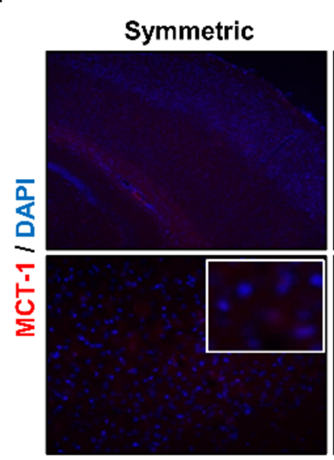

D

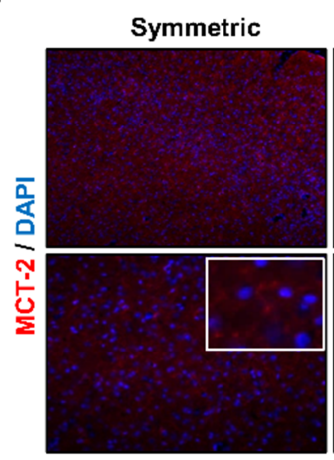

G

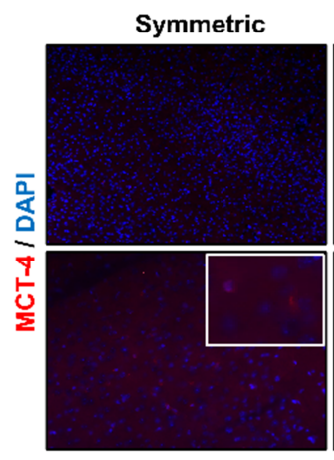

B

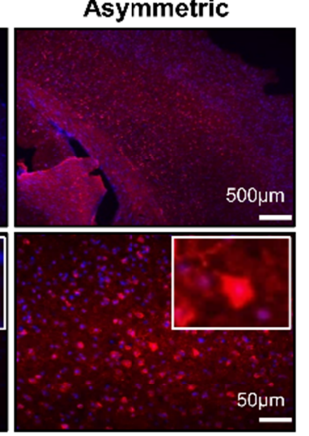

Asymmetric

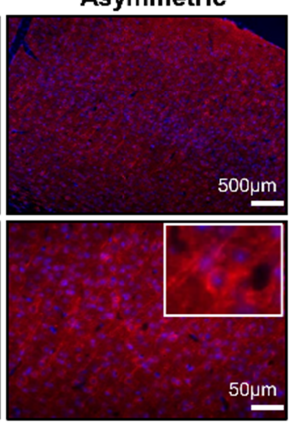

E
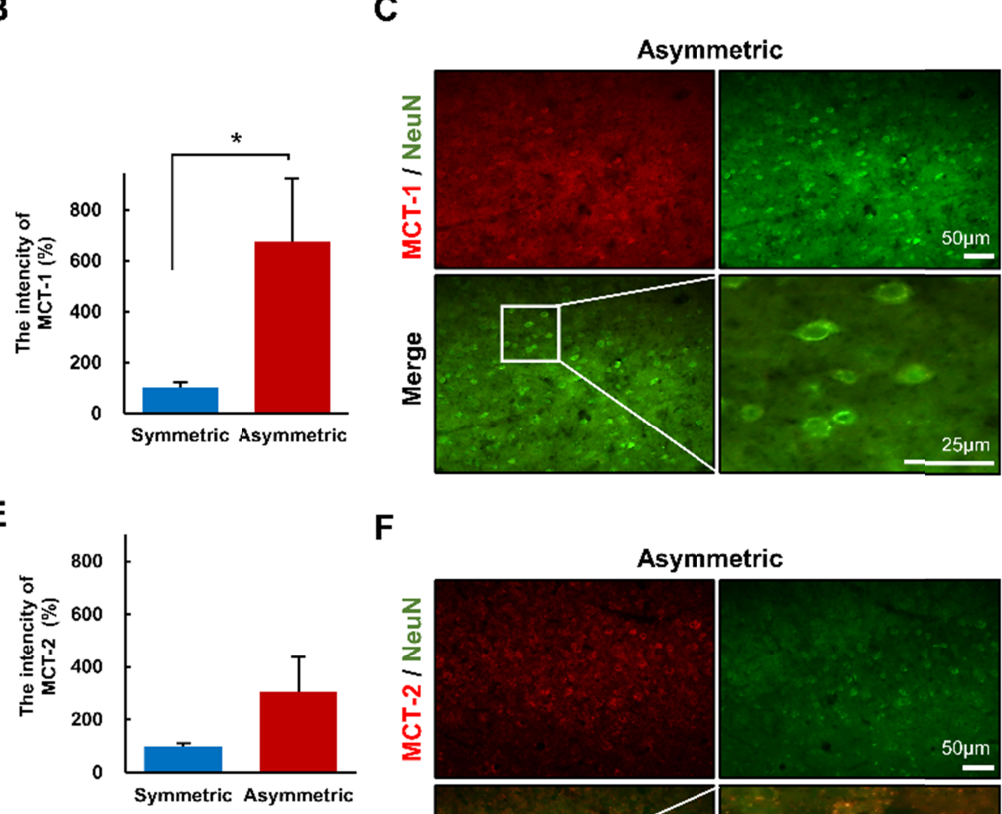

H

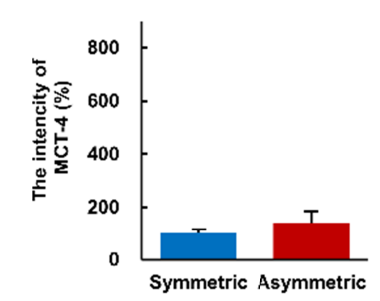

J

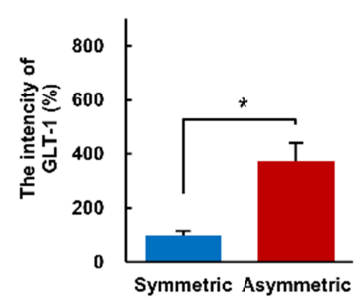

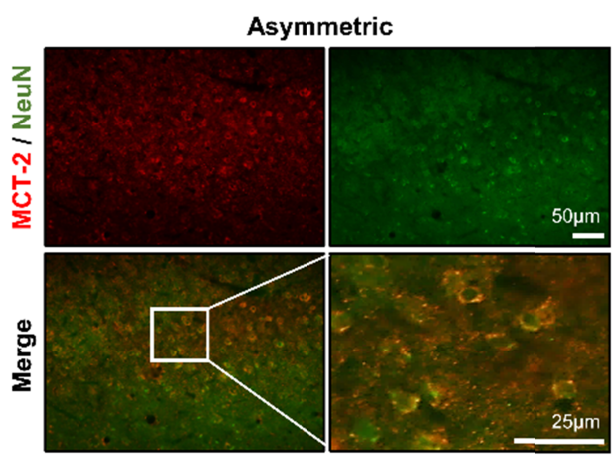

I

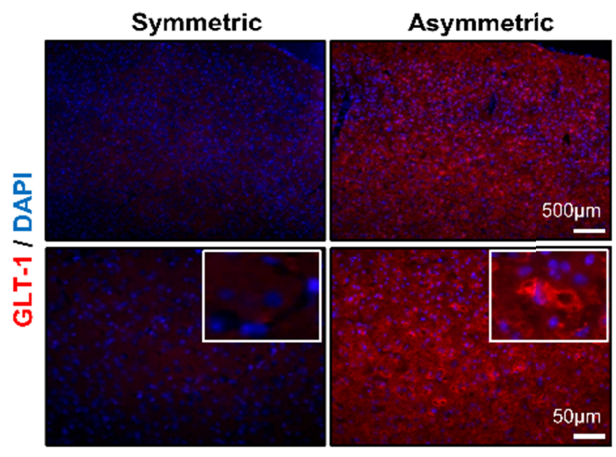

Fig. 6. Immunohistochemistry analysis of MCT-1, MCT-2, MCT-4, and GLT-1 in the cortex of the ipsilesional hemisphere of the UCCAO mouse brains (A) MCT-1 expression was observed to increase in the asymmetric group. (B) Intensity of MCT-1 significantly differed between groups ( $\mathrm{n}=6$ per each group, $\mathrm{p}<0.05$ ). (C) MCT-1 appeared to colocalize with neurons in the ischemic hemisphere of the asymmetric group. (D) MCT-2 expression tended to increase in the asymmetric group. (E) The difference did not reach statistical significance ( $\mathrm{n}=6$ per each group). (F) MCT-2 appeared to colocalize with neurons in the ischemic hemisphere of the asymmetric group. (G) MCT-4 expression was found along the vessels. (H) The expression did not differ between groups ( $\mathrm{n}=6$ ). (I) GLT-1 (red) expression was observed to increase in the asymmetric group. (J) The intensity of GLT-1 significantly differed between groups $(\mathrm{n}=4$ per each group, $\mathrm{p}<0.05$ ). No specific findings were observed in the contralateral hemisphere in the asymmetric brains. Data are expressed as the mean \pm standard error of the mean. GLT, glutamate transporter; MCT, monocarboxylate transporter; UCCAO, unilateral common carotid artery occlusion. ${ }^{*} \mathrm{p}<0.05 ; t$-test.

with GFAP, was upregulated in the right hemisphere of the asymmetric group, relative to the symmetric group (Fig. 5A 5D). Furthermore, astrocytes were more activated in the asymmetric group, relative to the symmetric group (Fig. 5E 5G). However, GLUT3 was similarly expressed in both groups (Fig. $5 \mathrm{H} \sim 5 \mathrm{~J}$ ). No specific findings were observed in the contralateral hemisphere of the asymmetric brains.

The expression levels of MCTs that carry lactates (end products of glycolysis) between astrocytes and neurons were presumed to be important for interpreting the high uptake of glucose in 
the more severe ischemic hemisphere. MCT-1 (Fig. 6A 6C) and MCT-2 (Fig. 6D 6F) appeared to be upregulated in the right hemisphere of the asymmetric group, relative to that of the symmetric group while MCT-4 (Fig. 6G 6H) did not look different between the groups. MCT- 1 and MCT-2 were expressed and colocalized with NeuN in the asymmetric group (Fig. 6C and 6F). GLT-1, a glutamate transporter, expression was evaluated because its upregulation might affect further glucose uptake in cerebral ischemia. GLT-1 was upregulated in the right hemisphere of the asymmetric group (Fig. 6I 6J)). The shape of this GLT-1 positive cells looked like astrocytes but not neurons in the hippocampus, where neurons are crowded (Supplementary Fig. 2). No specific findings were observed in the contralateral hemisphere in the asymmetric brains.

\section{DISCUSSION}

The results of the current study revealed some possible mechanisms regarding an asymmetric high uptake of FDG on PET at 7 days post-ischemia as shown in a subset of UCCAO mice. The increased glucose uptake was due to neither hyperperfusion nor higher neural activity, as blood flow decreased and neuronal death occurred in the high uptake hemisphere. In contrast, in this region, expression levels of GLUT1 were increased along the vessels, in conjunction with the activation of astrocytes. These results suggest that increased glucose uptake may be a compensatory mechanism that occurs in more severely ischemic brains, while most UCCAO mice only showed symmetric glucose uptake as this model is known to exhibit a relatively mild reduction of cerebral blood flow.

The first step for documenting the mechanism from our pilot finding of increased glucose uptake in UCCAO mice was to exclude a hyperperfusion phenomenon because the metabolic status evaluated by FDG PET can be coupled with perfusion status [9]. First, spontaneous reperfusion can be related to hyperperfusion. Spontaneous reperfusion occurs most frequently in a clinical setting at 7 days after acute ischemic stroke and might be associated with hyperperfusion $[2,6,19]$, which peaks within 2 weeks $[2,6]$. However, different from the clinical setting, a permanent occlusion is induced in the UCCAO model, which was used in the current study. The natural clinical course cannot be applied to our animal setting. Second, due to the small amount of energy storage within the brain, hyperemia can be induced in ischemic conditions [20]. In our experiments, FDG uptake increased 7 10 days after the UCCAO operation, suggesting a possible link between increased glucose uptake and hyperperfusion. However, we excluded this possibility, since laser Doppler flowmetry demonstrated reduced $\mathrm{CBF}$ in the hemisphere that showed increased glucose uptake on

\section{FDG PET.}

It has been reported that glucose uptake is reduced in the region of ischemia, following unilateral middle cerebral artery occlusion [3]. In rats with transient middle cerebral artery occlusion, CBF decreases by up to $80 \sim 90 \%$, relative to baseline [21]. This model consistently induces a definite cerebral infarction within the middle cerebral artery territory, with concomitant neuronal cell necrosis [22], which might result in decreased glucose uptake in the rat model $[3,4]$. In contrast, however, increased glucose uptake was observed in the ischemic hemisphere of the mice with UCCAO in our experiments. The difference might lie within the degree of cerebral ischemia; common carotid occlusion does not affect primary cerebral collateral circulation via the circle of Willis, thus, mild cerebral ischemia is induced, and definite neuronal necrosis is relatively rare. Typically, mice with permanent UCCAO exhibit reductions of approximately 20 30\% in CBF [11], as observed in mice in the symmetric group in the current study. In mice in the asymmetric group, CBF decreased further (by approximately $50 \sim 60 \%$ ) and induced an increase in glucose uptake in the ipsilateral cerebral hemisphere. This difference might have resulted from variations in the circle of Willis and in the effectiveness of collateral formation in arterial occlusion [23-26]. We attempted to distinguish these variations between groups; however, we could not identify the anterior communicating artery or measure its diameter due to small size of the structure in mice (Supplementary Fig. 3). Taken together, these findings indicate that moderate cerebral ischemia might induce increased glucose uptake in the ischemic hemisphere, whereas severe ischemia may result in evident cerebral infarction that reduces glucose uptake. Mild ischemia, a type of benign oligemia, may not change glucose metabolism in the brain.

The activation of astrocytes and glucose receptors along vessels in cellular level can explain the increased glucose uptake in vivo. First, astrocyte activation is involved. In a mild chronic ischemia model, similar to our UCCAO model, the proliferation of brain endothelial cells reached its peak at 4 days after hypoxia, and astrocyte activation began to occur 7 days after hypoxia [27]. In the current study, an asymmetric increase in glucose uptake was observed only at 7 and 10 days after UCCAO. In addition, it has been reported that astrocytes play a crucial role in the consumption of glucose in the brain [10,28-30]. Second, glucose uptake via GLUT1 may follow astrocyte activation. Among several types of glucose transporters, GLUT1, which plays a key role in glucose uptake in the brain, is expressed in the blood-brain barrier and in astrocytes [31-33]. Indeed, expression of GLUT1 in the cerebral vessels was upregulated in asymmetric brains after UCCAO.

The astrocyte-neuron lactate shuttle hypothesis may also partly 
explain our phenomenon. According to the theory, astrocytes produce lactate from glucose through glycolysis and supplies it to neurons as an energy source [10]. Astrocytic glycolysis may be stimulated by glutamate [10]. In our study, we found that expression of the glutamate transporter, GLT-1, was upregulated. In addition, MCTs that shuttle lactate between neurons and astrocytes were upregulated in asymmetric mice. Taken together, increased glucose uptake and increased expression levels of GLT-1 and MCTs in asymmetric mice may serve a major compensatory role in cerebral energy metabolism during hypoperfusion. However, this finding should be interpreted with caution because the activation of MCTs does not simply mean that lactate is transferred into neurons; the direction of the substrate was not proven in the current study.

Our study has some limitations. First, it was not determined why the two groups were different when they underwent the same UCCAO operation. Second, we could not evaluate the collateral patterns of mouse cerebral arteries due to their very small size. In addition, evaluations were limited due to a very low yield of the asymmetric glucose uptake phenomenon. In repeated experiments, it appeared only in a few cases among dozens of UC$\mathrm{CAO}$ mice. In one experiment, no mice showed the asymmetric phenomenon among 40 UCCAO mice. Nevertheless, this was a preliminary study; thus, further studies are needed, using prespecified cerebral blood flow groups with different levels of cerebral ischemia, to confirm our findings.

Based on our findings, we conclude that the in vivo high uptake of FDG in the hemisphere of mice with UCCAO was associated with more severe ischemia in terms of cerebral blood flow. The increased glucose uptake could be explained by glucose uptake via GLUT-1 and astrocytic activation at the cellular level.

\section{ACKNOWLEDGEMENTS}

This work was supported by the Basic-Clinical Translational Research fund of the Ajou University School of Medicine (J. S. Lee), and grants through the National Research Foundation of Korea (NRF) funded by the Ministry of Science, ICT \& Future Planning (NRF-2014R1A1A1008249, NRF-2018R1A2B6007094, J. S. Lee). The authors declare that the research was conducted in the absence of any commercial or financial relationships that could be construed as a potential conflict of interest.

\section{REFERENCES}

1. Astrup J, SiesjöBK, Symon L (1981) Thresholds in cerebral ischemia - the ischemic penumbra. Stroke 12:723-725.
2. Lenzi GL, Frackowiak RS, Jones T (1982) Cerebral oxygen metabolism and blood flow in human cerebral ischemic infarction. J Cereb Blood Flow Metab 2:321-335.

3. Balsara RD, Chapman SE, Sander IM, Donahue DL, Liepert L, Castellino FJ, Leevy WM (2014) Non-invasive imaging and analysis of cerebral ischemia in living rats using positron emission tomography with 18F-FDG. J Vis Exp (94):e51495.

4. Sobrado M, Delgado M, Fernández-Valle E, García-García L, Torres M, Sánchez-Prieto J, Vivancos J, Manzanares R, Moro MA, Pozo MA, Lizasoain I (2011) Longitudinal studies of ischemic penumbra by using 18F-FDG PET and MRI techniques in permanent and transient focal cerebral ischemia in rats. Neuroimage 57:45-54.

5. Powers WJ (2016) Cerebral blood flow and metabolism: regulation and pathophysiology in cerebrovascular disease. In: Stroke: pathophysiology, diagnosis, and management (Grotta JC, Albers G, Broderick JP, Kasner SE, Lo EH, Mendelow AD, Sacco RL, Wong KSL, eds), 6th ed. pp 28-46. Elsevier, Philaldephia.

6. Moretti JL, Defer G, Cinotti L, Cesaro P, Degos JD, Vigneron N, Ducassou D, Holman BL (1990) "Luxury perfusion" with 99mTc-HMPAO and 123I-IMP SPECT imaging during the subacute phase of stroke. Eur J Nucl Med 16:17-22.

7. Escartin C, Rouach N (2013) Astroglial networking contributes to neurometabolic coupling. Front Neuroenergetics 5:4.

8. Li B, Freeman RD (2015) Neurometabolic coupling between neural activity, glucose, and lactate in activated visual cortex. J Neurochem 135:742-754.

9. Bentourkia M, Bol A, Ivanoiu A, Labar D, Sibomana M, Coppens A, Michel C, Cosnard G, De Volder AG (2000) Comparison of regional cerebral blood flow and glucose metabolism in the normal brain: effect of aging. J Neurol Sci 181:19-28.

10. Bélanger M, Allaman I, Magistretti PJ (2011) Brain energy metabolism: focus on astrocyte-neuron metabolic cooperation. Cell Metab 14:724-738.

11. Yoshizaki K, Adachi K, Kataoka S, Watanabe A, Tabira T, Takahashi K, Wakita H (2008) Chronic cerebral hypoperfusion induced by right unilateral common carotid artery occlusion causes delayed white matter lesions and cognitive impairment in adult mice. Exp Neurol 210:585-591.

12. Lee JS, Im DS, An YS, Hong JM, Gwag BJ, Joo IS (2011) Chronic cerebral hypoperfusion in a mouse model of Alzheimer's disease: an additional contributing factor of cognitive impairment. Neurosci Lett 489:84-88.

13. Teune LK, Bartels AL, Leenders KL (2013) FDG- PET imaging in neurodegenerative brain diseases. In: Functional brain mapping and the endeavor to understand the working brain 
(Signorelli F, Chirchiglia D, eds), pp 463-476. IntechOpen, London.

14. Silva MD, Glaus C, Hesterman JY, Hoppin J, Puppa GH, Kazules T, Orcutt KM, Germino M, Immke D, Miller S (2013) Regional, kinetic [(18)F]FDG PET imaging of a unilateral Parkinsonian animal model. Am J Nucl Med Mol Imaging 3:129-141.

15. Xi W, Su D, Nie B, Yu Y, Shan B, Chen Q, Tian M, Zhang H (2013) 18F-FDG PET study reveals brain functional changes during attention in rats. J Nucl Med 54:1969-1973.

16. Segobin S, La Joie R, Ritz L, Beaunieux H, Desgranges B, Chételat G, Pitel AL, Eustache F (2015) FDG-PET contributions to the pathophysiology of memory impairment. Neuropsychol Rev 25:326-355.

17. Magistretti PJ, Pellerin L (1999) Cellular mechanisms of brain energy metabolism and their relevance to functional brain imaging. Philos Trans R Soc Lond B Biol Sci 354:1155-1163.

18. Yasaka M, Read SJ, O'Keefe GJ, Egan GF, Pointon O, McKay WJ, Donnan GA (1998) Positron emission tomography in ischaemic stroke: cerebral perfusion and metabolism after stroke onset. J Clin Neurosci 5:413-416.

19. Jørgensen HS, Sperling B, Nakayama H, Raaschou HO, Olsen TS (1994) Spontaneous reperfusion of cerebral infarcts in patients with acute stroke. Incidence, time course, and clinical outcome in the Copenhagen stroke study. Arch Neurol 51:865-873.

20. Petzold GC, Murthy VN (2011) Role of astrocytes in neurovascular coupling. Neuron 71:782-797.

21. Im DS, Jeon JW, Lee JS, Won SJ, Cho SI, Lee YB, Gwag BJ (2012) Role of the NMDA receptor and iron on free radical production and brain damage following transient middle cerebral artery occlusion. Brain Res 1455:114-123.

22. Yoon JS, Jo D, Lee HS, Yoo SW, Lee TY, Hwang WS, Choi JM, Kim E, Kim SS, Suh-Kim H (2018) Spatiotemporal protein atlas of cell death-related molecules in the rat MCAO stroke model. Exp Neurobiol 27:287-298.

23. Kitagawa K, Matsumoto M, Yang G, Mabuchi T, Yagita Y, Hori M, Yanagihara T (1998) Cerebral ischemia after bilateral ca- rotid artery occlusion and intraluminal suture occlusion in mice: evaluation of the patency of the posterior communicating artery.J Cereb Blood Flow Metab 18:570-579.

24. Hecht N, He J, Kremenetskaia I, Nieminen M, Vajkoczy P, Woitzik J (2012) Cerebral hemodynamic reserve and vascular remodeling in C57/BL6 mice are influenced by age. Stroke 43:3052-3062.

25. Cho KO, Kim SK, Kim SY (2017) Chronic cerebral hypoperfusion and plasticity of the posterior cerebral artery following permanent bilateral common carotid artery occlusion. Korean J Physiol Pharmacol 21:643-650.

26. Faber JE, Zhang H, Rzechorzek W, Dai KZ, Summers BT, Blazek C, Hedges SJ (2019) Genetic and environmental contributions to variation in the posterior communicating collaterals of the circle of Willis. Transl Stroke Res 10:189-203.

27. Li L, Welser JV, Dore-Duffy P, del Zoppo GJ, Lamanna JC, Milner R (2010) In the hypoxic central nervous system, endothelial cell proliferation is followed by astrocyte activation, proliferation, and increased expression of the alpha 6 beta 4 integrin and dystroglycan. Glia 58:1157-1167.

28. Magistretti PJ, Pellerin L (1996) The contribution of astrocytes to the 18F-2-deoxyglucose signal in PET activation studies. Mol Psychiatry 1:445-452.

29. Volterra A, Meldolesi J (2005) Astrocytes, from brain glue to communication elements: the revolution continues. Nat Rev Neurosci 6:626-640.

30. Zimmer ER, Parent MJ, Souza DG, Leuzy A, Lecrux C, Kim HI, Gauthier S, Pellerin L, Hamel E, Rosa-Neto P (2017) [18F] FDG PET signal is driven by astroglial glutamate transport. Nat Neurosci 20:393-395.

31. Jurcovicova J (2014) Glucose transport in brain - effect of inflammation. Endocr Regul 48:35-48.

32. Shah K, Desilva S, Abbruscato T (2012) The role of glucose transporters in brain disease: diabetes and Alzheimer's disease. Int J Mol Sci 13:12629-12655.

33. Patching SG (2015) Roles of facilitative glucose transporter GLUT1 in [18F]FDG positron emission tomography (PET) imaging of human diseases. J Diagn Imaging Ther 2:30-102. 